\title{
In utero and childhood DDT, DDE, PBDE and PCBs exposure and sex hormones in adolescent boys: The CHAMACOS study
}

\author{
Brenda Eskenazi ${ }^{\mathrm{a}, *}$, Stephen A. Rauch ${ }^{\mathrm{a}}$, Rachel Tenerelli ${ }^{\mathrm{a}}$, Karen Huen ${ }^{\mathrm{a}}$, Nina T. Holland ${ }^{\mathrm{a}}$, \\ Robert H. Lustig ${ }^{b}$, Katherine Kogut ${ }^{a}$, Asa Bradman ${ }^{a}$, Andreas Sjödin ${ }^{c}$, Kim G. Harley ${ }^{\text {a }}$ \\ a Center for Environmental Research and Children's Health (CERCH), School of Public Health, University of California, Berkeley, CA, USA \\ bepartment of Pediatrics, University of California, San Francisco, CA, USA \\ ${ }^{c}$ Division of Laboratory Sciences, National Center for Environmental Health, Centers for Disease Control and Prevention, Atlanta, GA, USA
}

\section{A R T I C L E I N F O}

\section{Article history:}

Received 24 August 2016

Received in revised form 30 October 2016

Accepted 2 November 2016

Chemical compounds studied in this article: p,p'- DDT (PubChem CID: 3036) p,p'-DDE (PubChem CID: 3035)

BDE-47 (PubChem ID: 95170)

BDE-99 (PubChem ID: 36159)

BDE-100 (PubChem ID: 154083)

BDE-153 (PubChem ID: 155166)

PCB- 28 (PubChem ID: 23448)

Testosterone (PubChem ID: 6013)

Keywords:

DDT

DDE

PCBs

Testosterone

Luteinizing hormone

Follicle stimulating hormone

Endocrine disruptors

Adolescence

Puberty

Prenatal

In utero

\begin{abstract}
A B S T R A C T
Dichlorodiphenyltrichloroethane (DDT), polybrominated diphenyl ether (PBDE) flame retardants, and polychlorinated biphenyls (PCBs) are believed to be endocrine-disrupting chemicals (EDCs) in humans and animals. The purpose of this study is to examine the relationship of in utero and childhood exposure to these purported EDCs and reproductive hormones in adolescent boys who participated in CHAMACOS, an ongoing birth cohort in California's Salinas Valley. We measured $o, p^{\prime}$ - and $p, p^{\prime}$-DDT, $p, p^{\prime}$-DDE, PBDEs and PCBs in serum collected from mothers during pregnancy or at delivery and from their sons at 9 years. We measured concentrations of follicle-stimulating hormone (FSH), luteinizing hormone (LH), and total testosterone $(\mathrm{T})$ from 234 of their sons at 12 years. In adjusted models, we found that a 10 -fold increase in maternal prenatal serum concentrations of BDE-153 was associated with a $22.2 \%$ increase (95\% CI: $1.0,47.9)$ in FSH, a $96.6 \%$ increase $(95 \%$ CI: $35.7,184.7)$ in $\mathrm{LH}$, and a $92.4 \%$ increase (95\% CI: 20.9, 206.2) increase in T. Similarly, BDE-100 concentrations were associated with increases in boys' LH levels. A 10fold increase in total prenatal $\Sigma$ PCBs was associated with a $64.5 \%$ increase $(95 \% \mathrm{CI}: 8.6,149.0)$ in $\mathrm{FSH}$, primarily driven by non- dioxin-like congeners. Boys' hormone levels were only marginally associated with prenatal DDT or DDE in primary models, but when boys' Tanner stage at age 12 was added to models, prenatal maternal DDT levels were associated with decreases in LH (adjusted percent change per 10-fold increase $=-18.5 \%, 95 \% \mathrm{CI}:-29.8,-5.4)$ and $\mathrm{T}$ (percent change $=-18.2 \%, 95 \% \mathrm{CI}$ : $-30.2,-4.2)$ and $\mathrm{DDE}$ with $\mathrm{LH}$ (percent change $=-18.3 \%, 95 \% \mathrm{CI}$ : $-32.9,-0.6$ ). Exposures measured in the children's serum at 9 years also showed associations between BDE-153 and $\Sigma$ PCBs. However, there is evidence that these associations appear to be mediated by child BMI. This study suggests associations on male hormones of 12 year old boys related to exposure to certain EDC exposure prenatally. The implications on future reproductive function in puberty and adulthood should be determined.
\end{abstract}

(c) 2016 Elsevier GmbH. All rights reserved.
Abbreviations: BMI, body mass index; CHAM1, CHAMACOS 1 (birth cohort); CHAM2, CHAMACOS 2 (enrolled at age 9 and followed prospectively); CV, coefficients of variation; DDT, dichlorodiphenyltrichloroet hane; DDE, dichlorodiphenyldichloroeth ylene; FSH, follicle-stimulating hormone; GM, geometric mean; GSD, gemoetric standard deviation; $\mathrm{kg} / \mathrm{m}^{2}$, Kilogram per meter squared; LH, luteinizing hormone; LLOQ, lower limit of quantification; LOD, limits of detection; $\mathrm{mIU} / \mathrm{ml}$, milli international units per milliliter; $\mathrm{ng} / \mathrm{dL}$, nanograms per deciliter; PBDE, polybrominated diphenyl ethers; $\mathrm{PCB}$, polychlorinated biphenyls; pg, picograms; T, total testosterone; TB, Technical Bulletin; US, United States; $\geq$, greater than or equal to; <, less than.

* Corresponding author at: Center for Environmental Research and Children's Health (CERCH), School of Public Health, University of California, Berkeley, 1995 University Ave Suite 265, Berkeley, CA USA.

E-mail address: eskenazi@berkeley.edu (B. Eskenazi).

\section{Introduction}

Chemicals such as dichlorodiphenyltrichloroethane (DDT), polybrominated diphenyl ethers (PBDEs), and polychlorinated biphenyls (PCBs), are hypothesized to be endocrine disruptors chemicals that mimic, block, or interfere with the body's natural hormones (Chiabotto et al., 2006; Diamanti-Kandarakis et al., 2009; Massart et al., 2006). DDT, an organochlorine pesticide, has not been used in the United States (US) since 1972 and in Mexico since 2000 (Blanco-Munoz et al., 2016); nonetheless, most US residents have measurable levels of the its metabolite, dichlorodiphenyldichloroethylene (DDE) (CDC, 2013). The commercial PBDE product penta-BDE (consisting of the congeners BDEs 
47, 99, 100, and 153) was added to foam-based furniture, textiles, and other consumer products until 2005, but continues to leach out from older household items. PCBs were used in the United States in dielectric and coolant fluids until 1979. These three chemical groups have long half-lives, are lipophilic, and bioaccumulate in the environment and in living organisms (SCPOP, 2016a; UNEP, 2001). They readily cross the placenta and reach the developing fetus (Vizcaino et al., 2014). Under the Stockholm Convention, PCBs and penta-BDE have been eliminated, and DDT use has been restricted to disease vector control (SCPOP, 2016b; UNEP, 2001). Although these chemicals are hypothesized to affect hormones, there are few human studies. Recent evidence suggests that age of pubertal onset in boys, defined by testicular enlargement (gonadarche) and the appearance of pubic hair (pubarche), may also be decreasing, and exposure to endocrine disruptors has been implicated (HermanGiddens et al., 2001; Herman-Giddens, 2006).

DDT compounds, such as the isomers $p, p^{\prime}$-DDT and $o, p^{\prime}$-DDT that are found in technical grade DDT, are known to be at least mildly estrogenic, and $p, p^{\prime}$ DDE inhibits androgens from binding to their receptors (Kelce et al., 1995). A study of 120 Akwesasne Mohawk boys (aged 10-17 years) reported that concurrent measurements of $p, p^{\prime}$-DDT were not associated with testosterone (T) levels, although $p, p^{\prime}$-DDE levels were positively but not significantly associated (Schell et al., 2014). The one previous study that examined the association of in utero exposure to DDT/DDE on the hormones of adolescent boys found no association between maternal prenatal levels during a time of peak use in the US and their sons' T levels at ages $10-17$ years $(n=260)$ (Gladen et al., 2004).

In estrogen receptor binding assays, lower-brominated PBDEs (e.g. BDE-28, -47 and -100 ) exhibit estrogenic activity (Dang et al., 2007; Meerts et al., 2001), while higher-brominated congeners (e.g. BDE-153 and -190) display anti-estrogenic properties (Meerts et al., 2001). The penta-BDE mixture also exhibits antiandrogenic activity in androgen receptor binding assays (Harju et al., 2007; Stoker et al., 2005). PBDEs have been found to be antiandrogenic in vivo in male rodent models, with studies of in utero BDE-99 exposure resulting in significant decreases in $\mathrm{T}$ in male offspring and a slight acceleration in onset of puberty (preputial separation) in low-dose males (Lilienthal et al., 2006). However, peripubertal penta-BDE exposure caused delayed preputial separation in male rodents (Stoker et al., 2004). No previous studies have examined the relationship in humans of in utero PBDE exposure on sex hormone levels, but significant inverse associations have been reported between penta-BDEs in house dust and FSH, LH, and free androgen index (molar ratio of total $\mathrm{T}$ to sex hormone-binding globulin) in a study of 24 men recruited from an infertility clinic (Meeker et al., 2009), but only the association with FSH persisted in the expanded sample of 62 men (Johnson et al., 2013).

PCB exposure studies have demonstrated conflicting relationships with male hormones in both animal (Wakui et al., 2010; Xiao et al., 2011; Xu et al., 2013; Zhu et al., 2013) and human studies. For example, in a cohort of boys with higher prenatal exposure to PCBs during the Yucheng incident in 1978-79 $(n=47)$ and unexposed controls $(n=49)$, exposed boys who were at puberty $(\geq 13$ years old) had lower T levels and higher estradiol and FSH levels (non-significant) compared to controls, but there was no difference in boys < 13 years; there were also no differences in LH levels (Hsu et al., 2005). Conversely, in a much larger study of the Faroe Islands birth cohort, PCBs in cord blood were inversely related to serum concentrations of T and LH in 14-year old boys ( $n=433$ ) (Grandjean et al., 2012).

In the present study, we determine the relationship between prenatal and childhood serum concentrations of DDT/DDE, pentaBDEs, and PCBs on the sex hormones of 12-year old boys participating in the Center for the Health Assessment of Mothers and Children of Salinas (CHAMACOS) birth cohort study. We hypothesize that early life exposure to EDCs will alter sex hormones of adolescent boys, although the direction of change is not apparent from prior literature.

\section{Methods}

\subsection{Study participants}

Pregnant women were recruited between October 1999 and October 2000 from community clinics. Women were eligible for participation if they were $\geq 18$ years old, $<20$ weeks gestation, Spanish- or English-speaking, qualified for low-income health insurance, and planned to deliver at the public hospital. A total of 601 women, known as the CHAMACOS 1 or CHAM1 cohort, enrolled in the study, of whom 526 were followed to the delivery of a liveborn, surviving singleton, of whom 263 were boys. The children were also assessed at multiple study visits approximately every 1 to 2 years, with 163 boys followed until age 12 . Detailed methods for CHAM1 are published elsewhere (Eskenazi et al., 2004, 2006). When the CHAM1 children were 9 years old (2009-2011), a second cohort of 305 9-year old children, CHAMACOS 2 (CHAM2), were recruited, 161 of whom were boys, with 149 followed to age 12. Eligibility criteria were similar to those for CHAM1. Mothers gave written informed consent; children gave verbal assent starting at age 7 and written assent at age 12 . All study activities were approved by the University of California at Berkeley Committee for the Protection of Human Subjects and the Centers for Disease Control (CDC) internal review board (IRB).

\subsection{Data collection}

CHAM1 women were interviewed twice during pregnancy (at around 13 and 26 weeks gestation), soon after delivery, and at each of the children's follow-up visits. Bilingual and bicultural interviewers conducted interviews in Spanish or English. During each interview, information was collected about family sociodemographic characteristics, maternal characteristics, and pregnancy and medical histories, as well as child developmental milestones, diet, and behavior. For CHAM2 mothers, data on pregnancy and the children's first 9 years were collected retrospectively at the child's 9-year visit. Children were interviewed privately at the 12 year old visit about their smoking and alcohol use, as well as other behaviors and habits. Children's height and weight were also measured at each of these visits, and body mass index (BMI, $\mathrm{kg} / \mathrm{m}^{2}$ ) was calculated.

Assessments of genital and pubic hair development were performed using the Tanner scale with stages from 1 (pre-puberty) to 5 (completed puberty) (Marshall and Tanner, 1970); highly trained research staff collected Tanner measurements at 9, 9.75, 10.5, 11.25, 12 , and 12.75 years. Quality control of Tanner staging was overseen by a pediatric endocrinologist.

\subsection{Measurement of DDT/DDE, PBDEs, and PCBs}

For CHAM1 mothers, we measured the concentrations of DDT/DDE $(n=83)$, PBDEs $(n=82)$, and PCBs $(n=86)$ in blood serum samples collected via venipuncture during pregnancy ( mean \pm standard deviation $=27.4 \pm 3.0$ weeks gestation) or at delivery. For CHAM1 and CHAM2 participants, DDT/DDE, PBDEs, and PCBs were measured in blood serum samples from the women and their children when the children were 9 years old.

Serum samples were immediately processed and stored at $-80^{\circ} \mathrm{C}$ at the University of California, Berkeley, School of Public Health Biorepository until being shipped on dry ice for analysis at the CDC (Atlanta, GA). p,p'-DDT, $p, p^{\prime}$-DDE and $o, p^{\prime}$-DDT, 10 PBDE congeners (BDEs 17, 28,47, 66, 85, 99, 100, 153, 154, and 183), 
and 34 PCB congeners (International Union for Pure and Applied Chemistry numbers $18,28,44,49,52,66,74,87,99,101,105$, $110,118,128,138,146,149,151,153,156,157,167,170,172$, $177,178,180,183,187,189,194,195,196,199,206$, and 209) were detected using gas chromatography isotope dilution high resolution mass spectrometry (GC-ID-HRMS). The limits of detection (LODs) for maternal samples were $0.8-6.7 \mathrm{ng} / \mathrm{g}$ lipid for DDT and DDE, $0.2-2.6 \mathrm{ng} / \mathrm{g}$ lipid for BDE-47, 0.2-0.7 ng/g for other PBDE congeners, and $0.008-2.14 \mathrm{ng} / \mathrm{g}$ lipid for PCBs. The LODs for child samples were 1.2-9.9 for DDT and DDE, 0.7-5.8 for BDE 47, 0.3-2.9 for other PBDE congeners, and 0.2-6.2 ng/g lipids for PCBs. For maternal samples taken at 9 years, the LODs were 0.6-9.75 for DDT and DDE, 0.7-5.8 for BDE-47, and 0.2-2.9 for the other PBDE congeners. Each run of 24 unknowns included quality control samples $(\mathrm{n}=3)$ and method blank samples $(\mathrm{n}=3)$. The analytical procedure has been published previously (Sjodin et al., 2008).

Concentrations are expressed on a serum lipid basis (nanograms/g lipids); total serum lipid concentrations were determined based on the measurement of triglycerides and total cholesterol using standard enzymatic procedures (Roche Chemicals, Indianapolis, IN), and the total lipid concentration was calculated based on an equation including the total cholesterol and triglyceride concentration (Phillips et al., 1989).

For 24 CHAM1 mothers in our final sample who lacked a prenatal blood sample but had blood collected at delivery, we estimated prenatal concentrations using predicted values from a univariate regression of delivery on prenatal concentrations. The correlations between prenatal and delivery exposure concentrations were all $\geq 0.95$, so other predictors were not considered necessary (all regression $r^{2}$ values were $\geq 0.9$ ) (Castorina et al., 2011). For both PBDEs and PCBs, the machine-read values were assigned for values less than the LOD if a signal was detected; and if no signal was detected, levels were imputed at random based on a log-normal probability distribution below the LOD via maximum likelihood estimation (Lubin et al., 2004).

For all CHAM2 children plus the 27 CHAM1 children whose mothers were missing prenatal or delivery measurements $(n=149)$, we predicted prenatal $p, p^{\prime}$-DDT or $p, p^{\prime}$-DDE concentrations using the levels in maternal and child blood taken at the 9-year visit in combination with other covariates (mothers' age, education, marital status, parity, and poverty status, duration of breastfeeding, and maternal weight and BMI both before pregnancy and when her child was 9 years old) (Verner et al., 2015). Models predicting the four primary prenatal penta-PBDE congeners $(n=142)$ were generated based on serum concentrations at the 9-year visit for mothers only, since inclusion of child's levels yielded a weaker predictive model (Verner et al., 2015). Prediction methods were developed using a Super Learner algorithm (Van der Laan et al., 2007), which yielded the following $\mathrm{r}^{2}$ values in the larger sample that these predictions were based on: $p, p^{\prime}-\mathrm{DDT}=0.95, p, p^{\prime}-\mathrm{DDE}=0.96$, $\mathrm{BDE}-47=0.79, \mathrm{BDE}-99=0.67, \mathrm{BDE}-100=0.80$, and BDE-153 $=0.85$ (Verner et al., 2015). We did not develop a prediction model for PCBs because of the number of different congeners that would need to be considered and the low detection frequency of most.

\subsection{Measurements of hormones}

Concentrations of LH, FSH, and T were measured in serum samples collected before 9 AM. Of the 312 boys who completed the 12-year visit, 234 (112 boys from CHAM1 and 122 boys from CHAM2) provided a blood sample of sufficient volume for hormone analyses. (One child was missing an FSH value due to an insufficient quantity of sample.) A total of 211 of these 234 boys had measurements of DDT/DDE, PBDEs, and PCBs from blood collected at their 9-year visit.
LH (lower limit of quantification $(\mathrm{LLOQ})=0.017 \mathrm{mIU} / \mathrm{ml}$ ) and FSH $(L L O Q=0.005 \mathrm{mIU} / \mathrm{ml})$ were determined by electrochemiluminescent assay. Blanks, repeats, and internal standards were included for quality assurance. The intra- and inter-assay coefficients of variation (CVs) for both assays were less than $6 \%$ and $7 \%$, respectively. T levels were analyzed using liquid chromatography with mass spectrometry detection after nonpolar solvent extraction; the LLOQ was $2.5 \mathrm{ng} / \mathrm{dL}$. Samples were processed with inclusion of water blanks, 5 assay control pools, and a 200 pg standard to assess accuracy and precision of the assay. The intra-assay $\mathrm{CV}$ was $<6 \%$ and the inter-assay $\mathrm{CV}$ was $<10 \%$. All assays were performed by Esoterix Laboratory Services (Calabasas Hills, CA).

\subsection{Statistical analysis}

Hormone and exposure variables all showed right-skewed distributions and were $\log _{10}$-transformed to more closely approximate a normal distribution. Thus, models are interpreted as the percent change in hormone levels per 10-fold increase in the concentration of the analyte(s). Because of the low detection for $o, p^{\prime}$-DDT (26.7\%), we restricted our analysis of DDT/DDE to $p, p^{\prime}$-DDT and $p, p$ '-DDE. We selected for statistical analysis the penta-PBDE congeners (BDE-47, $-99,-100$, and -153 ) since their detection frequencies were greater than $75 \%$. Penta-BDE congeners were analyzed individually and as a variable summing the four congeners. Only individual PCB congeners with a detection frequency greater than $75 \%$ (PCBs 18, 28, 44, 49, 52, 66, 74, 99, 101, 118, 138, 146, $153,156,170,180,187,194,196$, and 199 for maternal samples; and PCBs $28,118,138,153$, and 180 for child 9-year samples) were considered in statistical analyses. Because PCBs may have different modes of hormonal action, in maternal models we classified congeners based on dioxin-like (PCBs 118, 156, 170, 180) and nondioxin-like (PCBs 18, 28, 44, 49, 52, 66, 74, 99, 101, 138, 146, 153, $187,194,196$, and 199) activity (Van den Berg et al., 2006). In child serum samples, only five PCB congeners exhibited sufficient detection, and those were dominated by a single congener (PCB28); as a result, we did not divide child $\mathrm{PCB}$ concentrations into dioxin-like and non-dioxin-like categories.

Models with maternal DDT/DDE and PBDEs were run in two different ways: using measured concentrations only and using both measured and extrapolated maternal prenatal concentrations. We also constructed models using the measured levels of $p, p^{\prime}-\mathrm{DDT}, p, p^{\prime}-$ DDE, and PBDEs (BDE-47, 99, 100,153, and their sum) in child serum collected when the children were 9 years of age.

Covariates that were considered for inclusion included mothers' age at menarche and pre-pregnancy BMI; maternal age, race, years in the United States, and education at time of delivery; children's birthweight, gestational age at birth, birth order, and duration of exclusive breastfeeding; children's exact age, BMI, smoking, and alcohol use status at age 12 years; and family poverty status at each visit (see Table 1). Covariates were chosen based on a directed acyclic graph (see Supplemental Figs. 1 and 2) and included maternal education and family poverty at age 9 , as well as child age at hormone assessment and cohort (CHAM1 or 2) (if applicable). Models with maternal exposures also included maternal pre-pregnancy BMI. Child's age was treated as a continuous variable; family poverty, maternal education and pre-pregnancy BMI, and cohort were parameterized as categorical variables (see Table 1). Twentythree mothers in the CHAM2 cohort were missing pre-pregnancy BMI values, so we averaged the maternal BMI values at 9, 10.5, and 12-year visits and formed categories using those values $(<25$, $25-30$, and $>30 \mathrm{~kg} / \mathrm{m}^{2}$ ). We included Tanner genital development stage in sensitivity analyses in order to determine whether in utero chemical exposure was related to hormone levels within a given pubertal stage. Thirteen boys were missing Tanner data at 12 years. In sensitivity analyses, we included both maternal and child mea- 
Table 1

Demographics and medical characteristics of boys followed to age 12 years, CHAMACOS. 1999-2014.

\begin{tabular}{|c|c|}
\hline Maternal Characteristics & $\mathrm{N}(\%)$ \\
\hline \multicolumn{2}{|l|}{ Age at delivery, years } \\
\hline $18-24$ & $96(41.0)$ \\
\hline $25-29$ & $73(31.2)$ \\
\hline $30-34$ & $40(17.1)$ \\
\hline $35-45$ & $25(10.7)$ \\
\hline \multicolumn{2}{|l|}{ Education } \\
\hline$\leq 6$ th grade & $94(40.3)$ \\
\hline$\overline{7}-12$ th grade & $82(35.2)$ \\
\hline$\geq$ High school & $57(24.5)$ \\
\hline \multicolumn{2}{|l|}{ Race } \\
\hline Latino & $227(97.4)$ \\
\hline Other & $6(2.6)$ \\
\hline \multicolumn{2}{|l|}{ Country of birth } \\
\hline U.S. & $23(9.8)$ \\
\hline Other & $211(90.2)$ \\
\hline \multicolumn{2}{|l|}{ Years in the U.S. } \\
\hline$\leq 1$ & $55(23.5)$ \\
\hline $2-5$ & $53(22.7)$ \\
\hline $6-10$ & $61(26.1)$ \\
\hline $11+$ & $45(19.2)$ \\
\hline Entire life & $20(8.6)$ \\
\hline \multicolumn{2}{|c|}{ At or below the Census poverty line at 9 years postpartum } \\
\hline No & $63(26.9)$ \\
\hline Yes & $171(73.1)$ \\
\hline \multicolumn{2}{|l|}{ Parity } \\
\hline 0 & $77(32.9)$ \\
\hline 1 & $68(29.1)$ \\
\hline $2+$ & $89(38.0)$ \\
\hline \multicolumn{2}{|l|}{ Pre-pregnancy BMI, $\left(\mathrm{kg} / \mathrm{m}^{2}\right)$} \\
\hline$<18.5$ & $3(1.3)$ \\
\hline $18.5-24.9$ & $85(36.3)$ \\
\hline $25-29.9$ & $83(35.7)$ \\
\hline$\geq 30$ & $63(26.9)$ \\
\hline Age at menarche, years & \\
\hline$<12$ & $42(18.0)$ \\
\hline $12-13$ & $112(47.9)$ \\
\hline$>13$ & $80(34.2)$ \\
\hline Alcohol consumption durin & \\
\hline No & $202(86.7)$ \\
\hline Yes & $31(13.3)$ \\
\hline Smoked during pregnancy & \\
\hline No & $230(98.3)$ \\
\hline Yes & $4(1.7)$ \\
\hline Child Characteristics & $\mathrm{N}(\%)$ \\
\hline At Birth & \\
\hline Year of birth & \\
\hline 2000 & $73(31.2)$ \\
\hline 2001 & $102(43.6)$ \\
\hline 2002 & $59(25.2)$ \\
\hline Low birthweight $(<2500 \mathrm{~g})$ & \\
\hline No & $219(94)$ \\
\hline Yes & $14(6.0)$ \\
\hline Preterm birth ( $<37$ weeks) & \\
\hline No & $214(91.5)$ \\
\hline Yes & $20(8.5)$ \\
\hline Delivered by Cesarean Sect & \\
\hline No & $188(80.3)$ \\
\hline Yes & $46(19.7)$ \\
\hline Exclusively breastfed, mon & \\
\hline None & $18(7.9)$ \\
\hline$<2$ & $106(46.3)$ \\
\hline $2-4$ & $34(14.6)$ \\
\hline$>4$ & $71(31.0)$ \\
\hline At Age 12 & \\
\hline BMI percentiles & \\
\hline$<85$ th percentile & $95(41.1)$ \\
\hline 85-95th percentile & $48(20.8)$ \\
\hline$>95$ th percentile & $90(38.6)$ \\
\hline Tanner genital stage $2+$ & \\
\hline No & $25(11.3)$ \\
\hline Yes & $196(88.7)$ \\
\hline Tanner pubic hair stage $2+$ & \\
\hline No & $111(50)$ \\
\hline Yes & $111(50)$ \\
\hline Ever smoked & \\
\hline No & $226(96.6)$ \\
\hline Yes & $8(3.4)$ \\
\hline Ever tried alcohol & \\
\hline No & $195(83.3)$ \\
\hline Yes & 39 (16.7) \\
\hline
\end{tabular}

surements in the same model in order to assess their joint effects, although in some cases their correlation was high.

To test for a non-linear relationship between exposures and outcomes, we also ran a generalized additive model with a 3-df cubic spline for the exposure. If the model suggested evidence of non-linearity, we examined GAM residual plots and models with blood concentrations categorized by tertiles to assess the shape of the relationship. Models were also run including all 3 classes of chemicals ( $p, p^{\prime}$-DDT or $p, p^{\prime}$ - DDE, $\Sigma$ PBDEs, $\Sigma$ PCBs) in order to determine which class of compounds may have been driving observed associations. Possible interactions between the different classes of chemicals were examined using cross-product terms, with p-values below 0.1 considered statistically significant interactions.

In addition, in models of child and of maternal exposure, we examined mediation by the child BMI with a counterfactual approach, using indirect effects as evidence of mediation (Valeri and Vanderweele, 2013).

All models used robust standard errors based on the HuberWhite sandwich estimator to account for deviation from linear regression model assumptions (Huber, 1967; White, 1980). All analyses were completed with Stata version 13.1 (Stata Corporation, College Station, Texas).

\section{Results}

Most of the mothers were Latina (97.4\%) and born in Mexico (88.0\%), with approximately half being in the United States for less than five years at the time of the index birth (Table 1). About threequarters of the women had less than a high school education, with $40.3 \%$ having only a grade school education. The preterm birth rate in this sample was $8.5 \%$. Almost all the boys had been breastfed (92.1\%) with $31 \%$ exclusively breastfed for more than four months. At the time of the 12 -year follow-up, $20.8 \%$ of the boys were overweight and $38.1 \%$ were obese, and almost all boys (88.7\%) had reached Tanner genital stage 2 . Few of the boys had tried smoking (3.4\%) or alcohol (16.7\%) at the time of their 12-year visit. Compared to boys who dropped out before age 12 , those with hormone data at 12 years had older mothers, were breastfed longer, were less likely to be born by Cesarean section, and had marginally higher total maternal prenatal PCBs. No other baseline characteristics differed between those who were followed up and those who dropped out.

Table 2 shows the distribution of maternal serum concentrations for DDT/DDE, PBDEs, and PCBs. Median concentrations of $p, p^{\prime}$-DDT and $p, p^{\prime}$-DDE were $12.6 \mathrm{ng} / \mathrm{g}$ lipid (Interquartile Range $(\mathrm{IQR})=7.1,50.3)$ and $496.9 \mathrm{ng} / \mathrm{g}$ lipid $(\mathrm{IQR}=260.4,1621.6)$, respectively, including measured and extrapolated values. Median concentrations of measured samples only were very similar: $12.7 \mathrm{ng} / \mathrm{g}$ lipid (IQR $=6.8,57.2$ ) for $p, p^{\prime}$-DDT and $567.6 \mathrm{ng} / \mathrm{g}$ lipid $(\mathrm{IQR}=214.3,1622.2)$ for $p, p^{\prime}$-DDE, with $100 \%$ detection. The median of the sum of the four PBDE congeners (BDE-47, $-99,-100,-153$ ) was $24.3 \mathrm{ng} / \mathrm{g}$ lipid ( $\mathrm{IQR}=15.4,37.6)$, including both measured and extrapolated prenatal maternal levels; the preponderance of the sum was contributed by BDE-47. The PBDE concentrations were similar when we restricted the sample to those with measured levels (see Table 2), with nearly $100 \%$ detectable for each congener. The median of the sum of PCBs was $65.8 \mathrm{ng} / \mathrm{g}$ lipid $(\mathrm{IQR}=43.3$, 100.3 ) with $100 \%$ detection for at least one congener. Measured maternal serum concentrations of $p, p^{\prime}$ - DDT and $p, p^{\prime}$-DDE were highly correlated $(r=0.89, p<0.001)$, whereas these both showed a weak negative correlation with $\Sigma$ PBDE $(r=-0.16, p=0.14$ and $\mathrm{r}=-0.05, \mathrm{p}=0.66$, respectively $)$ and $\Sigma \mathrm{PCB}(\mathrm{r}=-0.14, \mathrm{p}=0.25$ and $r=-0.03, p=0.82$, respectively); maternal concentrations of $\Sigma$ PBDE and $\Sigma$ PCB had a weak positive correlation $(r=0.12, p=0.34)$.

Table 2 also shows the distribution of concentrations for DDT/DDE, PBDEs, and PCBs measured in the boys' sera at 9 
Table 2

Distribution and geometric means of concentrations of biological measures of environmental chemicals in maternal and child sera, CHAMACOS.

\begin{tabular}{|c|c|c|c|c|c|c|c|c|c|c|}
\hline \multirow[b]{2}{*}{ Exposure } & \multirow[b]{2}{*}{$\mathrm{n}$} & \multirow[b]{2}{*}{ \% Detected } & \multirow[b]{2}{*}{ GM (GSD) } & \multirow[b]{2}{*}{ Min } & \multicolumn{5}{|c|}{ Percentile } & \multirow[b]{2}{*}{ Max } \\
\hline & & & & & 10th & 25 th & 50 th & 75th & 90th & \\
\hline \multicolumn{11}{|c|}{ Maternal PBDEs, ng/g lipids (measured and extrapolated)a } \\
\hline$\Sigma$ PBDEs & 224 & & $25.1(2.2)$ & 2.6 & 11.3 & 15.4 & 24.3 & 37.6 & 60.7 & 860.6 \\
\hline $\mathrm{BDE}-47$ & 224 & & $14.9(2.3)$ & 0.5 & 6.1 & 9.4 & 14.7 & 23.5 & 36.5 & 514.8 \\
\hline BDE-99 & 224 & & $4.5(2.3)$ & 0.3 & 1.9 & 2.7 & 4.4 & 6.9 & 11.6 & 113.2 \\
\hline BDE-100 & 224 & & $2.8(2.2)$ & 0.4 & 1.2 & 1.7 & 2.6 & 4.4 & 7.8 & 85.2 \\
\hline BDE-153 & 224 & & $2.3(2.2)$ & 0.2 & 1.0 & 1.5 & 2.1 & 3.5 & 6.2 & 158.6 \\
\hline \multicolumn{11}{|c|}{ Maternal PBDEs, ng/g lipids (measured only) } \\
\hline$\Sigma$ PBDEs & 82 & $100 \%$ & $23.3(2.6)$ & 2.6 & 7.6 & 12.5 & 23.4 & 39.4 & 60.7 & 475.7 \\
\hline BDE-47 & 82 & $98.8 \%$ & $13.8(2.8)$ & 0.5 & 4.3 & 6.6 & 15.4 & 24.9 & 36.3 & 306.9 \\
\hline BDE-99 & 82 & $97.6 \%$ & $4.0(2.8)$ & 0.3 & 1.3 & 2.3 & 3.6 & 7.0 & 12.6 & 113.2 \\
\hline BDE-100 & 82 & $96.3 \%$ & $2.5(2.5)$ & 0.4 & 0.8 & 1.5 & 2.4 & 4.3 & 6.9 & 52.1 \\
\hline BDE-153 & 82 & $97.6 \%$ & $2.2(2.3)$ & 0.2 & 0.9 & 1.3 & 2.2 & 3.4 & 5.8 & 27.6 \\
\hline \multicolumn{11}{|c|}{ Child PBDEs, ng/g lipids (measured only) } \\
\hline$\Sigma$ PBDEs & 211 & $100 \%$ & $63.9(2.1)$ & 7.4 & 25 & 41.4 & 62.8 & 112.5 & 185.4 & 392.4 \\
\hline BDE-47 & 211 & $99.5 \%$ & $34.7(2.3)$ & 2.1 & 11.2 & 21.1 & 35.0 & 60.4 & 102.9 & 196.9 \\
\hline BDE-99 & 211 & $97.6 \%$ & $7.8(2.5)$ & 0.7 & 2.3 & 4.2 & 7.7 & 14.5 & 28.5 & 76.7 \\
\hline BDE-100 & 211 & $99.6 \%$ & $7.4(2.2)$ & 0.4 & 2.6 & 4.6 & 7.8 & 11.9 & 20.8 & 40.5 \\
\hline BDE-153 & 211 & $99.5 \%$ & $10.5(2.1)$ & 0.5 & 4.3 & 6.5 & 10.0 & 15.1 & 26.9 & 173.1 \\
\hline \multicolumn{11}{|c|}{ Maternal DDT and DDE, ng/g lipids (measured and extrapolated) } \\
\hline$p, p^{\prime}-\mathrm{DDT}$ & 232 & & $24.1(5.6)$ & 2.6 & 4.9 & 7.1 & 12.6 & 50.3 & 482.2 & 6678.4 \\
\hline$p, p^{\prime}-\mathrm{DDE}$ & 232 & & $683.8(3.8)$ & 34.8 & 181 & 260.4 & 496.9 & 1621.6 & 5336.6 & 34605.3 \\
\hline \multicolumn{11}{|c|}{ Maternal DDT and DDE, ng/g lipids (measured only) } \\
\hline$p, p^{\prime}-\mathrm{DDT}$ & 83 & $100 \%$ & $26.0(6.7)$ & 2.6 & 4.7 & 6.8 & 12.7 & 57.2 & 400.4 & 6678.4 \\
\hline$p, p^{\prime}-\mathrm{DDE}$ & 83 & $100 \%$ & $713.1(4.3)$ & 73.3 & 146.7 & 214.3 & 567.6 & 1622.2 & 8208.7 & 34605.3 \\
\hline \multicolumn{11}{|c|}{ Child DDT and DDE, ng/g lipids (measured only) ${ }^{c}$} \\
\hline$p, p^{\prime}-\mathrm{DDT}$ & 211 & $16.6 \%$ & $1.8(2.4)$ & 0.6 & 0.9 & 1.0 & 1.4 & 2.6 & 5.6 & 194.4 \\
\hline$p, p^{\prime}-\mathrm{DDE}$ & 210 & $100 \%$ & $173.0(3.1)$ & 21.3 & 51.5 & 79.5 & 137.3 & 295.9 & 898.7 & 12385 \\
\hline \multicolumn{11}{|c|}{ Maternal PCBs, ng/g lipids (measured only) } \\
\hline$\Sigma$ PCBs & 86 & $100 \%$ & $66.0(1.8)$ & 16.1 & 31.1 & 43.3 & 65.8 & 100.3 & 148.1 & 247.7 \\
\hline Dioxin-like & 86 & $100 \%$ & $5.0(2.0)$ & 0.5 & 2.3 & 3.6 & 5.1 & 7.5 & 11.0 & 32.1 \\
\hline Non-dioxin-like & 86 & $100 \%$ & $60.0(1.8)$ & 12.9 & 27.5 & 39.1 & 60.6 & 91.9 & 141.4 & 219.7 \\
\hline \multicolumn{11}{|c|}{ Child PCBs, ng/g lipids (measured only) } \\
\hline$\Sigma$ PCBs & 211 & $100 \%$ & $21.4(2.1)$ & 3.1 & 9.3 & 13.5 & 20.8 & 31.1 & 54.5 & 194.5 \\
\hline
\end{tabular}

a Measured in prenatal (i.e., from pregnancy or from delivery) serum or back- extrapolated from 9-year visit.

b Measured in prenatal serum.

c Measured in boys' sera at 9- year visit.

years of age. The median level of $p$, $p^{\prime}$-DDT in children was low (median $=1.4 \mathrm{ng} / \mathrm{g}$ lipid; $\mathrm{IQR}=1.0,2.6$ ) compared to mothers' ( median $=12.7 \mathrm{ng} / \mathrm{g}$ lipid, IQR $=6.8,57.2$ ), and only $16.6 \%$ of samples contained detectable levels. The median level of child $p, p^{\prime}$-DDE ( median $=137.3 \mathrm{ng} / \mathrm{g}$ lipid; $\mathrm{IQR}=79.5,295.9$ ) was less than a quarter of measured maternal levels ( median $=567.6 \mathrm{ng} / \mathrm{g}$ lipid; $\mathrm{IQR}=214.3$, 1622.2 ), but with $100 \%$ detection. The median of the child's $\Sigma$ PCB ( median $=20.8 \mathrm{ng} / \mathrm{g}$ lipid; $\mathrm{IQR}=13.5,31.1$ ) was less than a third of the mothers' levels (median $=65.8 \mathrm{ng} / \mathrm{g}$ lipid; $\mathrm{IQR}=43.3,100.3$ ). In contrast, the median of the $\Sigma$ PBDE congeners was more than twice as high in child than in maternal serum (median $=62.8 \mathrm{ng} / \mathrm{g}$ lipid; $\mathrm{IQR}=41.4,112.5$ ). Correlations between measured maternal prenatal and child 9-year $p, p$ '-DDT, $p$, $p$ '-DDE, $\Sigma$ PBDE, and $\Sigma$ PCB levels were $0.81(\mathrm{p}<0.001), 0.76(\mathrm{p}<0.001), 0.18(\mathrm{p}<0.13)$, and 0.18 $(\mathrm{p}<0.14)$, respectively.

Table 3 shows the serum levels of FSH, $\mathrm{LH}$, and testosterone in the CHAMACOS boys at 12 years old. The median concentration of each hormone was as follows: FSH was $2.5 \mathrm{mIU} / \mathrm{mL}$ serum $(\mathrm{IQR}=1.8,3.4)$, $\mathrm{LH}$ was $2.3 \mathrm{mIU}(\mathrm{IQR}=1.3,3.7)$, and $\mathrm{T}$ was $74.5 \mathrm{ng} / \mathrm{dL}$ $(\mathrm{IQR}=18,272)$.

Table 4 presents the adjusted models for DDT/DDE, PBDEs, and PCBs in maternal prenatal serum and hormone levels in the 12-year old boys. Maternal serum p,p'-DDT (measured only, or measured and extrapolated) were somewhat associated with decreases in the boys' LH and T, which became statistically significant when we adjusted for Tanner stage (see Table 4, Supplemental Table 1). Specifically, after controlling for Tanner stage, a 10-fold increase in maternal p,p'-DDT concentrations was associated with an $18.5 \%$ decrease in $\mathrm{LH}(95 \%$ confidence interval $(\mathrm{CI})=-29.8,-5.4)$ and an $18.2 \%$ decrease in $\mathrm{T}(95 \% \mathrm{C}=-30.2,-4.2)$ (See Supplemental Table
1). Similarly, a 10 -fold increase in $p$, $p^{\prime}$-DDE was related to an $18.3 \%$ decrease in $\mathrm{LH}(95 \% \mathrm{CI}=-32.9,-0.6)$, when we controlled for Tanner stage.

In contrast, prenatal maternal serum concentrations of $\Sigma$ PBDE (measured and extrapolated) were associated with an increase in the boys' LH levels (adjusted percent change per 10-fold increase $=51.0 \% ; 95 \% \mathrm{CI}=3.0,121.3$ ) but with no relationship with FSH or T (Table 4). For individual congeners, maternal concentrations of BDE-100 were associated with an increase in $\mathrm{LH}$ levels (adjusted percent change per 10 -fold increase $=75.1 \%$; $95 \%$ $\mathrm{CI}=21.8$, 151.6), while maternal BDE-153 concentrations were associated with increases in FSH (adjusted percent change per 10 -fold increase $=22.2 \%$; 95\% CI $=1.0,47.9$ ), $\mathrm{LH}$ (adjusted percent change per 10 -fold increase $=96.6 \%$; $95 \% \mathrm{CI}=35.7,184.7$ ) and $\mathrm{T}$ (adjusted percent change per 10 -fold increase $=92.4 \%$; $95 \% \mathrm{CI}=20.9,206.2$ ). When analysis was restricted to measured maternal levels only, results were similar except that maternal concentrations of BDE-100 were now also associated with an increase in boys' $\mathrm{T}$ levels (adjusted percent change per 10 -fold increase $=118.7 \%$; $95 \% \mathrm{CI}=10.6,332.2$ ), and maternal BDE-153 concentrations were no longer associated with the boys' FSH levels. Supplemental Table 1 shows that even after controlling for the boys' Tanner stage, significant positive associations were found between BDE-100 (measured and extrapolated) and LH (adjusted percent change per 10 -fold increase $=58.9 ; 95 \% \mathrm{CI}=5.0,140.4$ ) and between BDE-153 and LH (adjusted percent change per 10- fold increase $=79.0 ; 95 \% \mathrm{CI}=19.2,168.8)$ and $\mathrm{T}$ (adjusted percent change per 10 -fold increase $=56.0 ; 95 \% \mathrm{CI}=16.9,108.2$ ).

Similarly, maternal prenatal $\Sigma$ PCBs levels had positive relationships with hormone levels of the 12-year olds (Table 4) but were 
Table 3

Hormone levels (FSH, LH, and testosterone) in boys at age 12, CHAMACOS cohort, 2012-2014.

\begin{tabular}{|c|c|c|c|c|c|c|c|c|c|}
\hline \multirow[b]{2}{*}{ Outcome } & \multirow[b]{2}{*}{$\mathrm{N}$} & \multirow[b]{2}{*}{ GM (GSD) } & \multirow[b]{2}{*}{ Min } & \multicolumn{5}{|c|}{ Percentile } & \multirow[b]{2}{*}{ Max } \\
\hline & & & & $\overline{10 \text { th }}$ & 25 th & 50th & 75th & 90 th & \\
\hline FSH & 233 & $2.0(2.0)$ & 0.16 & 1.3 & 1.8 & 2.5 & 3.4 & 4.7 & 9.5 \\
\hline LH & 234 & $2.0(3.0)$ & 0.01 & 0.53 & 1.3 & 2.3 & 3.7 & 5.1 & 13.0 \\
\hline Testosterone & 234 & $69.0(4.0)$ & 4.3 & 9.4 & 18.0 & 74.5 & 272 & 422 & 780 \\
\hline
\end{tabular}

FSH and LH concentrations are in $\mathrm{mIU} / \mathrm{mL}$ serum. Testosterone concentrations are in ng/dL serum.

Table 4

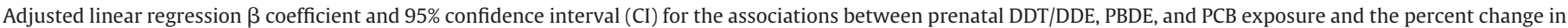
hormone levels of 12-year old boys, CHAMACOS.

\begin{tabular}{|c|c|c|c|c|}
\hline Exposure & $\mathrm{N}$ & FSH (\% change \& CI) & LH (\% change \& $\mathrm{CI})$ & Testosterone (\% change \& $\mathrm{CI}$ ) \\
\hline \multicolumn{5}{|c|}{ Maternal DDT and DDE (measured and extrapolated) } \\
\hline pp'-DDT & 232 & $-5.0(-13.0,3.7)$ & $-12.9(-26.0,2.4)$ & $-18.0(-34.9,3.3)$ \\
\hline pp'-DDE & 232 & $-9.2(-18.9,1.6)$ & $-12.9(-29.6,7.7)$ & $-21.1(-42.2,7.6)$ \\
\hline \multicolumn{5}{|c|}{ Maternal DDT and DDE (measured only) } \\
\hline pp'-DDT & 83 & $7.7(-6.0,23.5)$ & $-9.6(-28.7,14.5)$ & $-10.3(-36.0,25.7)$ \\
\hline pp'-DDE & 83 & $6.3(-11.1,27.2)$ & $-6.8(-34.1,31.6)$ & $-7.2(-42.4,49.3)$ \\
\hline \multicolumn{5}{|c|}{ Maternal PBDEs (measured and extrapolated) } \\
\hline$\Sigma$ PBDEs & 224 & $12.4(-7.5,36.5)$ & $51.0(3.0,121.3)^{*}$ & $36.2(-21.5,136.3)$ \\
\hline BDE-47 & 224 & $8.6(-9.6,30.6)$ & $37.4(-5.7,100.2)$ & $18.5(-32.0,106.7)$ \\
\hline BDE-99 & 224 & $9.8(-9.9,33.8)$ & $36.9(-4.5,96.0)$ & $31.1(-22.8,122.5)$ \\
\hline BDE-100 & 224 & $14.9(-5.7,40.0)$ & $75.1(21.8,151.6)^{*}$ & $55.6(-6.0,157.6)$ \\
\hline BDE-153 & 224 & $22.2(1.0,47.9)^{*}$ & $96.6(35.7,184.7)^{*}$ & $92.4(20.9,206.2)^{*}$ \\
\hline \multicolumn{5}{|c|}{ Maternal PBDEs (measured only) } \\
\hline$\Sigma$ PBDEs & 82 & $-0.1(-24.0,31.4)$ & $59.8(-11.6,188.9)$ & $94.2(-7.6,308.1)$ \\
\hline BDE-47 & 82 & $-0.3(-22.4,28.2)$ & $44.7(-18.3,156.3)$ & $66.9(-20.2,248.8)$ \\
\hline BDE-99 & 82 & $1.6(-21.9,32.1)$ & $48.4(-9.6,143.5)$ & $72.9(-10.3,233.3)$ \\
\hline BDE-100 & 82 & $-5.8(-28.9,24.9)$ & $84.0(8.7,211.5)^{*}$ & $118.7(10.6,332.2)^{*}$ \\
\hline BDE-153 & 82 & $0.2(-26.0,35.7)$ & $140.3(40.1,312.1)^{*}$ & $207.1(53.8,513.3)^{*}$ \\
\hline \multicolumn{5}{|c|}{ Maternal PCBs (measured only) } \\
\hline$\Sigma$ PCBs & 86 & $64.5(8.6,149.0)^{*}$ & $42.0(-45.1,267.3)$ & $153.9(-30.5,827.9)$ \\
\hline Dioxin-like & 86 & $9.1(-18.8,46.7)^{*}$ & $31.7(-32.0,155.2)$ & $84.6(-28.7,377.7)$ \\
\hline Non-dioxin-like & 86 & $63.3(9.3,143.9)^{*}$ & $33.7(-46.5,234.0)$ & $124.2(-37.3,702.0)$ \\
\hline
\end{tabular}

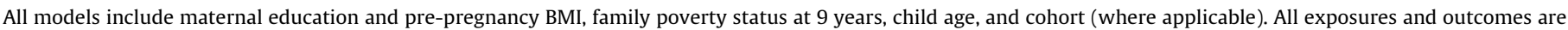
$\log 10$ - transformed; therefore, the results reported represent the percent change in hormones per 10-fold increase in chemical concentration (data not shown). * $\mathrm{p}<0.05$.

only significantly associated with FSH levels. Specifically, maternal prenatal $\Sigma$ PCB concentrations were related to a $64.5 \%$ increase in boys' FSH levels $(95 \% \mathrm{CI}=8.6,149.0)$. This relationship is driven by the non-dioxin-like PCBs (adjusted percent change per 10-fold increase $=63.3 \% ; 95 \% \mathrm{CI}=9.3,143.9$. The results controlling for Tanner stage were similar.

Although numbers were appreciably smaller $(n=69$ or 70 ), when we included the measured concentrations of $\Sigma$ PBDE, $\Sigma$ PCB and $p, p^{\prime}$-DDT or $p, p^{\prime}$-DDE in the same model, only $\Sigma$ PCB remained associated with elevated FSH (see Supplemental Table 2). We found no statistical interaction between different exposures when added to the above models.

There was no evidence of non-linearity in the relationships presented above and no evidence of mediation of child BMI on the association of maternal chemical concentrations and child hormone levels (data not shown).

As shown in Table 5, child BDE-153 levels at age 9 were associated with an increase in $\mathrm{LH}$ (adjusted percent change per 10-fold increase $=59.0 \% 95 \% \mathrm{CI}=8.6,132.6$ ) and $\mathrm{T}$ (adjusted percent change per 10 -fold increase $=149.1 \%$; $95 \% \mathrm{CI}=42.7,334.6$ ) and child $\Sigma \mathrm{PCB}$ concentrations with an increase in $\mathrm{T}$ at age 12 years (adjusted percent change per 10 -fold increase $=108.9 \%$; $95 \% \mathrm{CI}=7.1,307.2$ ). However, these associations appear to be primarily mediated by the child's BMI with no evidence of a direct effect of chemical concentrations on hormones. Specifically, child BMI mediated $42.0 \%$ of the association of child BDE-153 levels and LH, 30.3\%, of BDE153 and T, and $47.7 \%$ of $\Sigma$ PCB and T. No other associations were found between the child's chemical concentrations and hormone levels. Adding Tanner stage to these models did not appreciably change the results (see Supplemental Table 3). We found no statistical interaction between different exposures when added to the above models, and no evidence of non- linearity in the relationships presented above.

When both maternal and child chemical concentrations were included in the same model, the results are similar to those found in the above separate models.

\section{Discussion}

We examined the relationship of maternal prenatal DDT, DDE, PBDEs, and PCBs serum concentrations with the sex hormone levels of their sons at 12 years of age. We found that mothers' levels of DDT/DDE were somewhat inversely related to their sons' sex hormones in our primary models, and $p, p^{\prime}$-DDE became significantly inversely associated with LH, and $p, p^{\prime}$ - DDT with LH and T, when Tanner stage was considered in the model. We also found that $\mathrm{PPBDE}$ concentrations, specifically of BDE-100 and -153 , were associated with increased hormone levels (BDE-100 with LH and BDE- 153 with FSH, LH and T). Maternal $\Sigma$ PCB concentrations, specifically the non-dioxin-like PCBs, were associated with increased FSH. Any associations found between the chemical concentrations in the children's blood at 9 years and their hormone levels at 12 years appeared to be mediated by the child's BMI.

Although cross-sectional studies of primarily European or US adults (Cocco et al., 2004; Ferguson et al., 2012; Goncharov et al., 2009; Hagmar et al., 2001) have reported no association between DDT or DDE and sex hormones, serum $p, p^{\prime}$-DDE has been associated with lowered $\mathrm{T}$ in more highly-exposed populations such as Mexi- 
Table 5

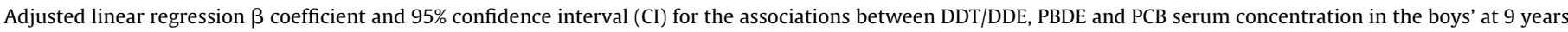
and the percent change in hormone levels at 12 years in the CHAMACOS cohort.

\begin{tabular}{|c|c|c|c|c|}
\hline Exposure & $\mathrm{N}$ & FSH (\% change \& CI) & $\mathrm{LH}(\%$ change \& $\mathrm{CI})$ & Testosterone (\% change \& $\mathrm{CI}$ ) \\
\hline$p, p^{\prime}-\mathrm{DDT}$ & 211 & $1.7(-13.9,20.1)$ & $-12.8(-36.3,19.5)$ & $-16.6(-47.1,31.5)$ \\
\hline$p, p^{\prime}-\mathrm{DDE}$ & 210 & $-4.3(-18.1,11.8)$ & $5.8(-24.9,49.0)$ & $7.5(-29.6,64.1)$ \\
\hline$\Sigma$ PBDEs & 211 & $0.3(-21.2,27.6)$ & $21.6(-29.1,108.4)$ & $40.4(-26.2,167.2)$ \\
\hline BDE-47 & 211 & $0.9(-18.9,25.5)$ & $14.3(-31.5,90.8)$ & $19.6(-33.5,115.1)$ \\
\hline BDE-99 & 211 & $-0.7(-17.9,20.1)$ & $9.1(-30.6,71.7)$ & $4.3(-37.5,74.3)$ \\
\hline BDE-100 & 211 & $0.1(-19.4,24.5)$ & $13.0(-31.2,85.6)$ & $18.1(-37.1,121.6)$ \\
\hline BDE-153 & 211 & $8.0(-10.7,30.6)$ & $59.0(8.6,132.6)^{*}, \dagger$ & $149.1(42.7,334.6)^{*}$ \\
\hline$\Sigma$ PCBs & 211 & $8.8(-14.1,37.8)$ & $26.8(-28.0,123.3)$ & $108.9(7.1,307.2)^{*}$ \\
\hline
\end{tabular}

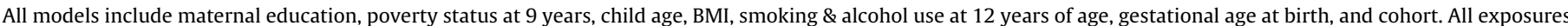
and outcomes are log10-ransformed; therefore, the results reported represent the percent change in hormones per 10 -fold increase in chemical concentration. $\mathrm{p}<0.05$.

$\dagger$ Association mediated by child BMI: $42.0 \%$ of total association between LH and BDE-153, 30.3\% of association of testosterone and BDE- 153 , and $47.7 \%$ of association between testosterone and $\mathrm{SPCBs}$.

can flower growers (Blanco-Munoz et al., 2012), residents of a rural Brazilian village where a chemical plant had been located (Freire et al., 2014), and a small sample of highly-exposed 16-28 year old Mexican men (Ayotte et al., 2001). In addition, Martin et al. found a decrease in $\mathrm{T}$ levels and free androgen index in African-American farmers, but only in those in the highest $10^{\text {th }}$ percentile of $p, p^{\prime}-D D E$ levels (2002). In contrast to the above findings, a relatively small study of South-African malaria vector- control workers $(n=47)$ who were highly-exposed (Dalvie et al., 2004) reported significant positive relationships between $p, p$ '-DDT and both estradiol and T, but not with FSH or LH; they reported no significant relationships with $p, p$ '-DDE. The only previous study examining the relationship of maternal prenatal exposure to DDT/DDE and adolescent hormones reported null findings (Gladen et al., 2004). Similar to some of the cross-sectional studies of men (Ayotte et al., 2001; Blanco-Munoz et al., 2012; Freire et al., 2014; Martin et al., 2002), we find some evidence of an inverse association of DDT/DDE and male hormones in the adolescents that are only statistically significant when we controlled for Tanner stage (see Supplemental Table 1), suggesting that within a given stage of puberty there may an inverse relationship with hormone levels. Diminished secretion of T can result in failure of gonadal function (hypogonadism), such that males fail to produce normal numbers of sperm (Dandona and Rosenberg, 2010). Thus, if the observed relationship continues into adulthood, a reduction in fertility may be noted.

We report, in this first study of in utero PBDE exposure and male adolescent hormones, that maternal blood concentrations of the longer half-life congeners BDE-100 and - 153 were associated with increases in some of the male hormones. Increased male hormones could result in earlier onset of puberty in these boys (possibly mediated via alteration of the hypothalamic-pituitary-gonadal axis). LH (which acts on Leydig cells in the testes to produce T) and FSH (which acts on Sertoli cells in the testes to initiate spermatogenesis) work synergistically and are generally elevated in male precocious puberty (Blondell et al., 1999; Dandona and Rosenberg 2010). However, there are few previous studies in humans to support our findings and results in animals are conflicting. For example, in animal studies, prenatal administration of BDE-99 at environmentally-relevant doses resulted in adult male offspring with smaller tested and epidydimus, and lower sperm production, but no effect on LH or T (Kuriyama et al., 2005), whereas another study found decreased estrogen and $\mathrm{T}$ and nonsignificantly earlier onset of puberty (Lilienthal et al., 2006), and yet another study found that PBDE-710 (a mixture of tetra- and penta-BDE) stimulated $\mathrm{T}$ secretion in rat Leydig cells (Wang et al., 2011). A previous cross-sectional study of men found inverse or no association with the same male hormones examined in the present study (Johnson et al., 2013; Meeker et al., 2009).
PCBs are mixtures of congeners with varying modes of action (Wolff et al., 1997); some act like dioxins on the aryl hydrocarbon receptor, and others are non-dioxin-like compounds, acting primarily as neurotoxicants by affecting the hypothalamic-pituitary-gonadal axis (Dandona and Rosenberg, 2010). Our finding that maternal prenatal serum concentrations of PCBs were positively associated with FSH in adolescents, particularly of non-dioxin-like congeners, shows mixed consistency with results of previous studies, possibly due to varying mixes of PCB congeners. PCB cord levels showed an inverse association with $\mathrm{T}$ and LH in 14-year old boys from the Faroe Islands birth cohort study (Grandjean et al., 2012). Their exposure was predominantly from consumption of whale blubber and to non-dioxin-like PCBs, for which we found positive associations with sex hormones. The nonsignificant findings in the Yucheng cohort, in which adolescents were exposed primarily to dioxin-like PCBs and dibenzofurans following a rice oil contamination, are more in line with the lack of association we found between dioxin-like PCBs and adolescent male reproductive hormones (Hsu et al., 2005). The overall levels of exposure in both the Faroe Islands and Yucheng studies are likely considerably higher than in our population, which had lower levels than the general US population participating in NHANES (CDC, 2005). The similarity in our findings from in utero exposure to PBDEs and to $\mathrm{PCBs}$ is noteworthy, given their resemblance in structure (Siddiqi et al., 2003).

While we found associations between male hormones in the 12-year olds and childhood (at 9-years) concentrations of BDE-153 (LH and T) and total PCBs (T only), these associations appear to be mediated by child BMI. We previously reported a positive relationship with body mass in these CHAMACOS boys at 7-years of age of maternal prenatal BDE-153 concentrations, but an inverse relationship of child BDE-153 concentrations (Erkin-Cakmak et al., 2015). Taken together, our results suggest a complex interrelationship between childhood PBDE levels, especially for the more lipophilic compounds, and body mass, pubertal onset, and male sex hormones (Chevrier, 2013).

The strengths of the present study are its unique longitudinal design, the use of prenatal as well as child blood chemical concentrations with a wide range of exposure, and extensive covariate information for both mothers and children. In addition, the population is relatively homogenous with regard to both race and social class, which potentially reduces the influence of unmeasured confounding. Furthermore, all boys were 12 years old, and we have considered Tanner staging in the analysis. However, the study is limited in that we use back-extrapolated exposure measures to estimate in utero exposure to DDT/DDE and PBDEs for some of the cohort, although results were similar in most cases when restricting the analysis to the group with measured levels. In utero concentrations of PBDEs and PCBs were similar or lower that the general US 
population's; because the maternal DDT/DDE levels were relatively high due to most mothers' recent immigration from Mexico, results on DDT/DDE may not be generalizable to the general US population. In addition, we did not measure some hydroxy-BDEs that may be more biologically active (Siddiqi et al., 2003), nor the highlybrominated compounds of PBDEs such as BDE-209, although they likely represent a small portion of the total PBDE body burden (Rose et al., 2010).

In conclusion, in this prospective follow-up study, we found that maternal prenatal blood concentrations of PCBs, PBDEs and DDT/DDE were associated with some alterations in serum hormone concentrations in adolescent boys, particularly for the higher congener PBDEs and the non-dioxin-like PCBs. Any associations of childhood blood concentrations of these endocrine-disrupting chemicals and sex hormones are likely mediated by associations of exposure with child BMI. Thus, these results suggest endocrine disruption due to exposures that occurred prenatally. Although the implications on future testicular and reproductive function in puberty and in adulthood are not known at this time, we are currently evaluating the relationship with in utero exposure to these endocrine disruptors and timing and tempo of puberty. Given the paucity of studies examining in utero exposures and male reproductive hormones and the inconsistencies in findings across the few existing studies, more research is needed to confirm our results and to determine whether the purported effects of these endocrine disruptors persist into adulthood, when hormone levels may be more stable.

\section{Grant information}

This publication was made possible by research supported by grant numbers: RD 83171001 and RD 826709 from the U.S. Environmental Protection Agency (US EPA), and PO1 ES009605 and RO1 ES015572 from NIEHS. The contents of this publication are solely the authors' responsibility and do not necessarily represent the official views of the NIEHS, NIH, EPA or the Centers for Disease Control and Prevention.

\section{Statementof financial interest}

The authors of this manuscript do not have any financial conflict of interest with any parties that were involved in this study.

\section{Disclaimer}

The findings and conclusions in this report are those of the authors and do not necessarily represent the official position of the Centers for Disease Control and Prevention.

\section{Acknowledgements}

We acknowledge the CHAMACOS phlebotomists, Norma Morga, Kimberley Parra and Celina Trujillo, as well as other field staff, students, community partners, and participants and families; the biorepository staff for their assistance in specimen management; and colleagues at CDC for specimen analysis. I would like to thank Drs. Fraser Gaspar and Marc-Andre Verner for their contribution to the back-extrapolation models of CHAMACOS in utero concentrations.

\section{Appendix A. Supplementary data}

Supplementary data associated with this article can be found, in the online version, at http://dx.doi.org/10.1016/j.ijheh.2016.11. 001.

\section{References}

Ayotte, P., Giroux, S., Dewailly, E., Hernandez Avila, M., Farias, P., Danis, R., et al., 2001. DDT spraying for malaria control and reproductive function in Mexican men. Epidemiology 12, 366-367.

Blanco-Munoz, J., Lacasana, M., Aguilar-Garduno, C., Rodriguez-Barranco, M. Bassol, S., Cebrian, M.E., et al., 2012. Effect of exposure to p,p'-DDE on male hormone profile in Mexican flower growers. Occup. Environ. Med. 69, 5-11.

Blanco- Munoz, J., Lacasana, M., Lopez-Flores, I., Rodriguez-Barranco, M., Gonzalez-Alzaga, B., Bassol, S., et al., 2016. Association between organochlorine pesticide exposure and thyroid hormones in floriculture workers. Environ. Res. 150, 357-363.

Blondell, R.D., Foster, M.B., Dave, K.C., 1999. Disorders of puberty. Am. Fam. Phys, 60, 209-218.

CDC, 2005. Third national report on human exposure to environmental chemicals.: 475 .

CDC, 2013. National Biomonitoring Program. Factsheet: Dichlorodiphenyltrichloroethane (DDT). U.S. Department of Health and Human Services, Centers for Disease Control and Prevention, Hyattsville, MD, Available: http://www.cdc.gov/biomonitoring/DDT_FactSheet. html (accessed June 2016).

Castorina, R., Bradman, A., Sjodin, A., Fenster, L., Jones, R.S., Harley, K.G., et al., 2011. Determinants of serum polybrominated diphenyl ether (PBDE) levels among pregnant women in the CHAMACOS cohort. Environ. Sci. Technol. 45, 6553-6560.

Chevrier, J., 2013. Invited commentary: maternal plasma polybrominated diphenyl ethers and thyroid hormones-challenges and opportunities. Am. J. Epidemiol. $178,714-719$.

Chiabotto, P., Costante, L., de Sanctis, C., 2006. Premature thelarche and environmental pollutants. Minerva Med. 97, 277-285.

Cocco, P., Loviselli, A., Fadda, D., Ibba, A., Melis, M., Oppo, A., et al., 2004. Serum sex hormones in men occupationally exposed to dichloro-diphenyl-trichloro ethane (DDT) as young adults. J. Endocrinol. 182, 391-397.

Dalvie, M.A., Myers, J.E., Lou Thompson, Lou, Dyer, S., Robins, T.G., Omar, S., et al., 2004. The hormonal effects of long-term DDT exposure on malaria vector-control workers in Limpopo Province, South Africa. Environ. Res. 96 $9-19$

Dandona, P., Rosenberg, M.T., 2010. A practical guide to male hypogonadism in the primary care setting. Int. J. Clin. Pract. 64, 682-696.

Dang, V.H., Choi, K.C., Jeung, E.B., 2007. Tetrabromodiphenyl ether (BDE 47) evokes estrogenicity and calbindin-D9k expression through an estrogen receptor-mediated pathway in the uterus of immature rats. Toxicol. Sci. 97 504-511.

Diamanti-Kandarakis, E., Bourguignon, J.P., Giudice, L.C., Hauser, R., Prins, G.S., Soto, A.M., et al., 2009. Endocrine-disrupting chemicals: an Endocrine Society scientific statement. Endocr. Rev. 30, 293-342.

Erkin-Cakmak, A., Harley, K.G., Chevrier, J., Bradman, A., Kogut, K., Huen, K., et al., 2015. In utero and childhood polybrominated diphenyl ether exposures and body mass at age 7 years: the CHAMACOS study. Environ. Health Perspect. 123, 636-642.

Eskenazi, B., Harley, K., Bradman, A., Weltzien, E., Jewell, N.P., Barr, D.B., et al., 2004. Association of in utero organophosphate pesticide exposure and fetal growth and length of gestation in an agricultural population. Environ. Health Perspect. $112,1116-1124$.

Eskenazi, B., Marks, A.R., Bradman, A., Fenster, L., Johnson, C., Barr, D.B., et al., 2006. In utero exposure to dichlorodiphenyltrichloroethane (DDT) and dichlorodiphenyldichloroethylene (DDE) and neurodevelopment among young Mexican American children. Pediatrics 118, 233-241.

Ferguson, K.K., Hauser, R., Altshul, L., Meeker, J.D., 2012. Serum concentrations of p, p'-DDE, HCB, PCBs and reproductive hormones among men of reproductive age. Reprod. Toxicol. (Elmsford, NY) 34, 429-435.

Freire, C., Koifman, R.J., Sarcinelli, P.N., Rosa, A.C., Clapauch, R., Koifman, S., 2014 Association between serum levels of organochlorine pesticides and sex hormones in adults living in a heavily contaminated area in Brazil. Int. J. Hyg. Environ. Health 217, 370-378.

Gladen, B.C., Klebanoff, M.A., Hediger, M.L., Katz, S.H., Barr, D.B., Davis, M.D., et al., 2004. Prenatal DDT exposure in relation to anthropometric and pubertal measures in adolescent males. Environ. Health Perspect. 112, 1761-1767.

Goncharov, A., Rej, R., Negoita, S., Schymura, M., Santiago-Rivera, A., Morse, G., et al., 2009. Lower serum testosterone associated with elevated polychlorinated biphenyl concentrations in Native American men. Environ. Health Perspect. 117, 1454-1460.

Grandjean, P., Gronlund, C., Kjaer, I.M., Jensen, T.K., Sorensen, N., Andersson, A.M., et al., 2012. Reproductive hormone profile and pubertal development in 14-year-old boys prenatally exposed to polychlorinated biphenyls. Reprod. Toxicol. (Elmsford, NY) 34, 498-503.

Hagmar, L., Bjork, J., Sjodin, A., Bergman, A., Erfurth, E.M., 2001. Plasma levels of persistent organohalogens and hormone levels in adult male humans. Arch. Environ. Health 56, 138-143.

Harju, M., Hamers, T., Kamstra, J.H., Sonneveld, E., Boon, J.P., Tysklind, M., et al., 2007. Quantitative structure-activity relationship modeling on in vitro endocrine effects and metabolic stability involving 26 selected brominated flame retardants. Environ. Toxicol. Chem. 26, 816-826.

Herman-Giddens, M.E., Wang, L., Koch, G., 2001. Secondary sexual characteristics in boys: estimates from the national health and nutrition examination survey III, 1988-1994. Arch. Pediatr. Adolesc. Med. 155, 1022-1028. 
Herman-Giddens, M.E., 2006. Recent data on pubertal milestones in United States children: the secular trend toward earlier development. Int. J. Androl. 29, 241-246, discussion 286-290.

Hsu, P.C., Lai, T.J., Guo, N.W., Lambert, G.H., Guo, Y.L., 2005. Serum hormones in boys prenatally exposed to polychlorinated biphenyls and dibenzofurans. J. Toxicol. Environ. Health A 68, 1447-1456.

Huber, P.J., 1967. The behavior of maximum likelihood estimates under nonstandard conditions. Proceedings of the Fifth Berkeley Symposium on Mathematical Statistics and Probability, Vol. 1. University of California Press, Berkeley, CA, pp. 221-233.

Johnson, P.I., Stapleton, H.M., Mukherjee, B., Hauser, R., Meeker, J.D., 2013. Associations between brominated flame retardants in house dust and hormone levels in men. Sci. Total Environ. 445-446, 177-184.

Kelce, W.R., Stone, C.R., Laws, S.C., Gray, L.E., Kemppainen, J.A., Wilson, E.M., 1995 Persistent DDT metabolite p,p'-DDE is a potent androgen receptor antagonist. Nature 375, 581-585.

Kuriyama, S.N., Talsness, C.E., Grote, K., Chahoud, I., 2005. Developmental exposure to low dose PBDE 99: effects on male fertility and neurobehavior in rat offspring. Environ. Health Perspect. 113, 149-154.

Lilienthal, H., Hack, A., Roth-Harer, A., Grande, S.W., Talsness, C.E., 2006. Effects of developmental exposure to 2,2,4,4,5-pentabromodiphenyl ether (PBDE-99) on sex steroids, sexual development, and sexually dimorphic behavior in rats. Environ. Health Perspect. 114, 194-201.

Lubin, J.H., Colt, J.S., Camann, D., Davis, S., Cerhan, J.R., Severson, R.K., et al., 2004. Epidemiologic evaluation of measurement data in the presence of detection limits. Environ. Health Perspect. 112, 1691-1696.

Marshall, W.A., Tanner, J.M., 1970. Variations in the pattern of pubertal changes in boys. Arch. Dis. Child. 45, 13-23.

Martin Jr., S.A., Harlow, S.D., Sowers, M.F., Longnecker, M.P., Garabrant, D., Shore, D.L., et al., 2002. DDT metabolite and androgens in African-American farmers. Epidemiology 13, 454-458.

Massart, F., Parrino, R., Seppia, P., Federico, G., Saggese, G., 2006. How do environmental estrogen disruptors induce precocious puberty? Minerva Pediatr. 58, 247-254.

Meeker, J.D., Johnson, P.I., Camann, D., Hauser, R., 2009. Polybrominated diphenyl ether (PBDE) concentrations in house dust are related to hormone levels in men. Sci. Total Environ. 407, 3425-3429.

Meerts, I.A., Letcher, R.J., Hoving, S., Marsh, G., Bergman, A., Lemmen, J.G., et al., 2001. In vitro estrogenicity of polybrominated diphenyl ethers, hydroxylated PDBEs, and polybrominated bisphenol A compounds. Environ. Health Perspect. 109, 399-407.

Phillips, D.L., Pirkle, J.L., Burse, V.W., Bernert Jr., J.T., Henderson, L.O., Needham, L.L., 1989. Chlorinated hydrocarbon levels in human serum: effects of fasting and feeding. Arch. Environ. Contam. Toxicol. 18, 495-500.

Rose, M., Bennett, D.H., Bergman, A., Fangstrom, B., Pessah, I.N., Hertz-Picciotto, I., 2010. PBDEs in 2-5 year-old children from California and associations with diet and indoor environment. Environ. Sci. Technol. 44, 2648-2653.

Stockholm Convention on Persistent Organic Pollutants (SCPOP), 2016. Listing of POPs in the Stockholm Convention. Available: http://chm.pops.int/ TheCo nvention/ThePOPs/ListingofPOPs/tabid/2509/Default. aspx [accessed July 2016].

Stockholm Convention on Persistent Organic Pollutants (SCPOP), 2016. The new POPs under the Stockholm Convention. Available: http://chm.pops.int/ TheConve ntion/ThePOPs/TheNewPOPs/tabid/2511/Default.aspx [accessed July 2016].

Schell, L.M., Gallo, M.V., Deane, G.D., Nelder, K.R., DeCaprio, A.P., Jacobs, A., et al., 2014. Relationships of polychlorinated biphenyls and dichlorodiphenyldichloroethylene (p,p'- DDE) with testosterone levels in adolescent males. Environ. Health Perspect. 122, 304-309.
Siddiqi, M.A., Laessig, R.H., Reed, K.D., 2003. Polybrominated diphenyl ethers (PBDEs): new pollutants-old diseases. Clin. Med. Res. 1, 281-290.

Sjodin, A., Wong, L.Y., Jones, R.S., Park, A., Zhang, Y., Hodge, C., et al., 2008. Serum concentrations of polybrominated diphenyl ethers (PBDEs) and polybrominated biphenyl (PBB) in the United States population: 2003-2004. Environ. Sci. Technol. 42, 1377-1384.

Stoker, T.E., Laws, S.C., Crofton, K.M., Hedge, J.M., Ferrell, J.M., Cooper, R.L., 2004 Assessment of DE-71, a commercial polybrominated diphenyl ether (PBDE) mixture, in the EDSP male and female pubertal protocols. Toxicol. Sci. 78, $144-155$.

Stoker, T.E., Cooper, R.L., Lambright, C.S., Wilson, V.S., Furr, J., Gray, L.E., 2005 In vivo and in vitro anti-androgenic effects of DE-71, a commercial polybrominated diphenyl ether (PBDE) mixture. Toxicol. Appl. Pharmacol. 207 78-88.

United Nations Environment Programme (UNEP), 2001. Final Act of the Conference of Plenipotentiaries on the Stockholm Convention on Persistent Organic Pollutants.

Valeri, L., Vanderweele, T.J., 2013. Mediation analysis allowing for exposure-mediator interactions and causal interpretation: theoretical assumptions and implementation with SAS and SPSS macros. Psychol. Methods 18, 137-150.

Van den Berg, M., Birnbaum, L.S., Denison, M., De Vito, M., Farland, W., Feeley, M., et al., 2006. The 2005 World Health Organization reevaluation of human and Mammalian toxic equivalency factors for dioxins and dioxin-like compounds. Toxicol. Sci. 93, 223-241.

Van der Laan, M.J., Polley, E.C., Hubbard, A.E., 2007. Super learner. Stat. Appl. Genet. Mol. Biol. 6, Article25.

Verner, M.A., Gaspar, F.W., Chevrier, J., Gunier, R.B., Sjodin, A., Bradman, A., et al., 2015. Increasing sample size in prospective birth cohorts: back-extrapolating prenatal levels of persistent organic pollutants in newly enrolled children. Environ. Sci. Technol. 49, 3940-3948.

Vizcaino, E., Grimalt, J.O., Glomstad, B., Fernandez-Somoano, A., Tardon, A., 2014 Gestational weight gain and exposure of newborns to persistent organic pollutants. Environ. Health Perspect. 122, 873-879.

Wakui, S., Muto, T., Motohashi, M., Kobayashi, Y., Suzuki, Y., Takahashi, H., et al., 2010. Testicular spermiation failure in rats exposed prenatally to $3,3^{\prime}, 4,4^{\prime}, 5-$ pentachlorobiphenyl. J. Toxicol. Sci. 35, 757-765.

Wang, K.L., Hsia, S.M., Mao, I.F., Chen, M.L., Wang, S.W., Wang, P.S., 2011. Effects of polybrominated diphenyl ethers on steroidogenesis in rat Leydig cells. Hum. Reprod. 26, 2209-2217.

White, H., 1980. A heteroskedasticity-consistent covariance matrix estimator and a direct test for heteroskedasticity. Econometrica 48, 817-830.

Wolff, M.S., Camann, D., Gammon, M., Stellman, S.D., 1997. Proposed PCB congener groupings for epidemiological studies. Environ. Health Perspect. 105, 13-14.

Xiao, W., Zhang, J., Liang, J., Zhu, H., Zhou, Z., Wu, Q., 2011. Adverse effects of neonatal exposure to 3,3',4,4',5,5'-hexachlorobiphenyl on hormone levels and testicular function in male Sprague-Dawley rats. Environ. Toxicol. 26, 657-668.

$\mathrm{Xu}, \mathrm{C} ., \mathrm{C}$ Chai, J., Qian, Y., 2013. [Effect of in utero and lactational exposure to a binary mixture of 3,3',4,4', 5- pentachlorobiphenyl (PCB126) and 2,3',4,4',5pentachlorobiphenyl (PCB118) on reproductive function in male rat offsprings]. Wei Sheng Yan Jiu 42, 758-763.

Zhu, Y., Mapuskar, K.A., Marek, R.F., Xu, W., Lehmler, H.J., Robertson, L.W., et al., 2013. A new player in environmentally induced oxidative stress: polychlorinated biphenyl congener, 3,3'-dichlorobiphenyl (PCB11). Toxicol. Sci. 136, 39-50. 